\title{
Structure of the Shigella flexneri podophage HRP29
}

\author{
Sundharraman Subramanian and Kristin Parent
}

Michigan State University, East Lansing, Michigan, United States

Short tailed phages (Podoviridae) are understudied compared to their long tailed phage counterparts, the Myoviridae and Siphoviridae. Podophages in Salmonella (P22), E. coli (T7), and Shigella (Sf6) have been structurally characterized and used as model systems to investigate capsid structure, morphogenesis, and stages of infection. However, many aspects of these processes are still unknown for a majority of environmental phage isolates. Recent interest in phage hunting activities has expanded the diversity and repertoire of phage isolates, and also provide new systems to investigate. As part of our phage hunting exercises to isolate Shigella flexneri phages from the environment, we have identified phages mostly belonging to the Myoviridae and Siphoviridae family [1,2], but to date only one short tailed, lytic podophage (HRP29) has been isolated [2].

The genome sequence of HRP29 has only $\sim 10 \%$ average nucleotide identity (ANI) to any known bacteriophage genome. In addition, the nearest family members of HRP29 which include phiKDA1 (host: E. cloacae) and KP34 (host: K. pneumoniae), have few biological details recorded. Efficiency of plating assays of HRP29 with $S$. flexneri mutants lacking known phage membrane protein receptors, show that HRP29 does not use the same receptors as the other podophages Sf6, T7 or P22. Although the structural genes of HRP29 follow a similar order to T7, it encodes a full-length Sf6-like tailspike protein rather than a true T7-like tail fiber. Single particle cryo-EM analysis of HRP29 was carried out and a icosahedrallyaveraged cryo-EM map of resolution of $3.8 \AA$ A was obtained. A homology model of HRP29 capsid protein was placed in the map, which revealed similarities to T7 capsid structure. The cryo-EM map also shows the presence of a decoration like protein on the surface of the HRP29 capsid, the role and identity of this protein is yet to be identified. 2D-class averages of HRP29 tail region have shown that HRP29 possesses a hybrid tail, combining both T7- and P22-like elements. HRP29 tail possesses a central tail tube similar to T7 but possesses a Sf6-like tailspike. Taken together, these data suggest that studying HRP29's mechanism of infection may reveal a novel mechanism of phage-host interactions, act as a framework for future studies of similar phages and enabling comparison to other podophages.

\section{References}

1. Doore SM, Schrad JR, Dean WF, Dover JA, Parent KN. 2018. Shigella Phages Isolated during a Dysentery Outbreak Reveal Uncommon Structures and Broad Species Diversity. J. Virol. 92(8):e0211717

2. Doore SM, Schrad JR, Perrett HR, Schrad KP, Dean WF, Parent KN. 2019. A cornucopia of Shigella phages from the Cornhusker State. Virology 538:45-52 